\title{
Effect of Varying Solvents on Structural Properties of Annealed PVDF-Trfe Thin Films
}

\author{
A.N. Arshad ${ }^{1}$, M.N. Sarip ${ }^{2}$, M. Rusop ${ }^{3}$, E.Z. Engku Zaharah ${ }^{1}$, M.D. Rozana ${ }^{1 *}$ \\ ${ }^{1}$ Faculty of Applied Sciences, Universiti Teknologi MARA, 40450 Shah Alam, Selangor, Malaysia \\ ${ }^{2}$ Faculty of Applied Sciences, Universiti Teknologi MARA, 02000 Arau, Perlis, Malaysia \\ ${ }^{3}$ NANO-SciTech (NST), Institute of Science (IOS), Universiti Teknologi MARA, 40450 Shah Alam, Selangor, Malaysia \\ *Corresponding author E-mail: rozanadahan@gmail.com
}

\begin{abstract}
PVDF-TrFE (70:30 mol\%) powders were dissolved individually in three polar solvents of Diethyl Carbonate (DEC), Methyl Ethyl Ketone (MEK) and N-, N-, Dimethylformamide (DMF). The solution was spin coated and formed PVDF-TrFE films of $250 \mathrm{~nm}$ thickness; UN DEC, UN MEK and UN DMF films. PVDF-TrFE powders were found to readily dissolve in DMF solvent as indicated by Hansen solubility parameters $\left(\delta_{t}\right)$. Hansen parameter showed small difference in reading $\left(\delta_{t}=1.7\right)$ for PVDF-TrFE and DMF, and low relative energy difference (RED) value. All films were annealed at $120^{\circ} \mathrm{C}$ (AN DEC, AN MEK, AN DMF). The AN DMF film showed high presence of polar $\beta$-phase crystals as evident from the ATR-FTIR spectrum. This is given by the significant peaks at 848,1190 , and 1290 $\mathrm{cm}^{-1}$. The trend is consistent with XRD pattern, where relative increment of cps value (53\%) at $19.2^{\circ}(2 \theta)$ is observed. This further supports the high degree of $\beta$-phase crystals observed in AN DMF film.
\end{abstract}

Keywords: PVDF-TrFE; Hansen Solubility Parameter; ATR-FTIR; XRD; Solvent;

\section{Introduction}

Polyvinylidene fluoride (PVDF) and its copolymers are widely used as polymeric materials for memory and storage devices due to their excellent dielectric properties [1-3]. PVDF is a semicrystalline polymer with complex structure consisting of 4 crystalline phases; $\alpha-, \beta-, \delta$-, and $\gamma$-crystals. Amongst these crystals, the $\alpha$ - and $\beta$-crystal dominate the crystal phases in PVDF. The $\beta$ crystal is a polar-phase crystal with large spontaneous polarization caused by the trans- (TTTT) conformation. Meanwhile, the $\alpha$ phase crystal has a trans-gauche conformation (TGTG) which neutralizes the dipolar polarization (zero net polarization) and thus not favourable for dielectric application.

In comparison, copolymers of PVDF, such as PVDF-TrFE exhibits Curie temperature (Tc) in the range of 55 to $128{ }^{\circ} \mathrm{C}$, depending on the percentage compositions of $\operatorname{TrFE}[4,5]$. This is the basis for the non-linear dielectric response of PVDF-TrFE, also known as ferroelectric property. Recent studies reported PVDF-TrFE with $30 \mathrm{~mol} \%$ TrFE will crystallize simply to $\beta$-crystals due to the thermodynamic stability $[4,6,7]$. Numerous studies are still being carried out to further extent the degree of $\beta$-phase crystals in PVDF-TrFE due to the need for high performance dielectric films used in memory devices and digital applications.

The typical method for enhancing the polar phases of PVDF-TrFE are typically by varying of annealing temperatures on PVDFTrFE, incorporation of nanoparticles in PVDF-TrFE or the use of polar solvents $[2,8,9]$. When complete dissolution takes place, solvent molecules overcome the intermolecular adhesion between molecules. Complete dissolution is possible when the charge or polarity of solute and solvent are comparable. This caused detachment of the molecules; and miscibility will follow. Hildebrand and Hansen solubility parameters can be used to determine the polarity for solvent and the compatibility between solvents and solutes. Hansen's parameter $\left(\partial_{\mathrm{T}}\right)$ is defined as the summation of dispersion force $\left(\partial_{\mathrm{d}}\right)$, polar interactions force $\left(\partial_{\mathrm{p}}\right)$ and hydrogen bonding force $\left(\partial_{\mathrm{h}}{ }^{2}\right)$ [10-12]. The equation for Hansen's parameters is as follow:

$$
\partial_{T}=\partial_{d}^{2}+\partial_{p}^{2}+\partial_{h}^{2}
$$

By using Hansen's parameters, the solubility parameters for Methyl Ethyl Ketone (MEK), N-,N-, Dimethylformamide (DMF) and Diethyl Carbonate are determined. The favourable solvent for complete dissolution of PVDF-TrFE (70:30 mol.\%) can be obtained. The effect of these solvents on their structural properties will be investigated.

\section{Experimental Procedure}

All materials were used as purchased; PVDF-TrFE (70:30 mol\%) (powder from PiezoTech, France), N-, N-, Dimethylformamide (DMF, 99.5\%, from Systerm), Methyl Ethyl Ketone (MEK from Sigma Aldrich) and Diethyl Carbonate (DEC from Systerm). PVDF-TrFE powders are dispersed at concentration of $30 \mathrm{~g} / \mathrm{L}$ in DMF, MEK and DEC solvents individually. The solution is stirred for 24 hours prior to deposition on spin coated aluminium-glass (al-glass) substrates rotated at $1500 \mathrm{rpm}$. These films were then annealed at $120^{\circ} \mathrm{C}$ for 1 hour in a vacuum oven. The annealing temperature for PVDF-TrFE was optimized at $120^{\circ} \mathrm{C}$ as established from previous study [13].

All films were scanned (ATR-FTIR Perkin Elmer with nickel filter) from wavenumber $2000 \mathrm{~cm}^{-1}$ to $600 \mathrm{~cm}^{-1}$ and functional groups were identified. The X-ray Diffraction (XRD) pattern 
(X'Pert Powder, PANalytical) at scanning angle $5^{\circ}$ to $80^{\circ}(2 \theta)$ was used to determine the crystallinity of the film. The data was re-plotted with X'pert Highscore Plus software; and peak observed will indicate the presence of a particular type of crystal phase in the film. Atomic Force Micrscopy (Park System) with scan area of $10 \mu \mathrm{m} \times 10 \mu \mathrm{m}$ were used to investigate the surface morphology of the film.

Hansen's solubility parameter and relative energy difference (RED) values for both PVDF-TrFE and solvents were determined and compared. Field Emission Scanning Electron Microscopy (FESEM) was used to observe the morphology of the optimized PVDF-TrFE thin films.

\section{Result and Discussions}

\subsection{ATR-FTIR}

Fig. 1 shows the ATR-FTIR spectra for un-annealed and annealed PVDF-TrFE thins films dissolved in DEC. Three intense bands of 848,1290 and $1400 \mathrm{~cm}^{-1}$ were observed, which indicates the formation of $\beta$-crystals in PVDF-TrFE [14]. The bands at 848 and $1290 \mathrm{~cm}^{-1}$ are assigned to $-\mathrm{CF}_{2}$ stretching mode coupled with the symmetric vibration of $-\mathrm{C}-\mathrm{C}$ - and bending vibration from the carbon backbone, whilst the band at $1400 \mathrm{~cm}^{-1}$ are assigned to the $\mathrm{CH}_{2}$ of PVDF-TrFE with wagging vibration coupled with the asymmetric stretching vibration of the carbon chain (-C-C-). Similar trends were observed for UN MEK and AN MEK (Fig. 2).

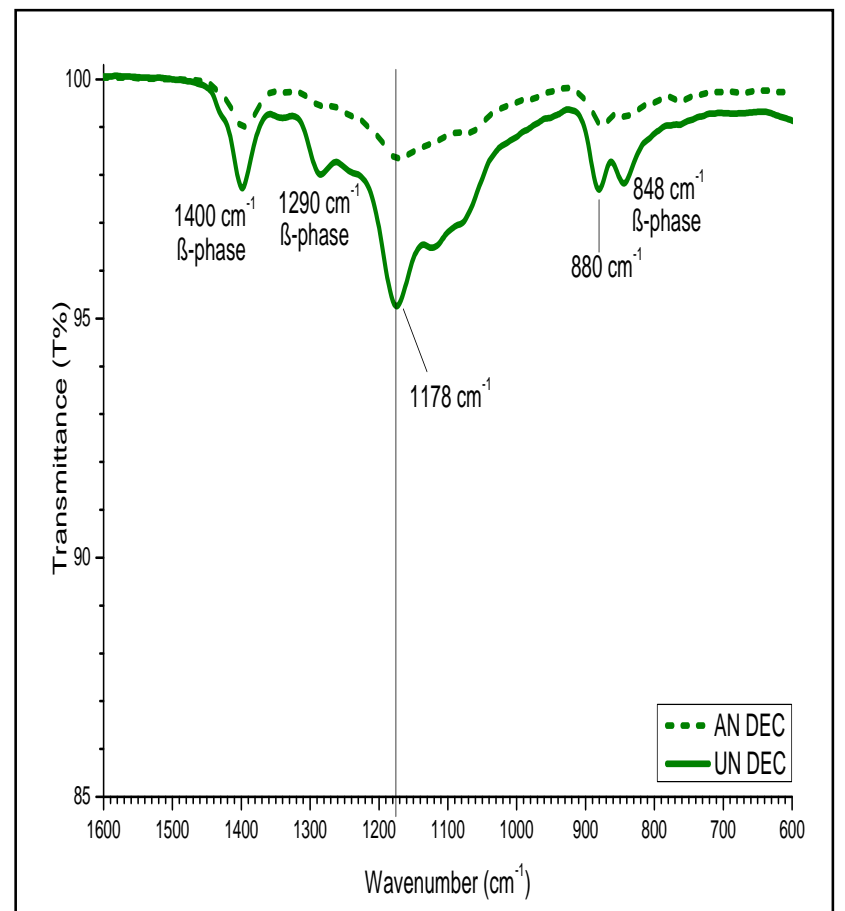

Fig. 1: ATR-FTIR spectra for the Un-annealed and annealed PVDF-TrFE thin film dissolved in DEC solvent.

At band $1172 \mathrm{~cm}^{-1}$, a peak was observed for UN DEC, AN DEC (Fig. 1) and AN MEK (Fig. 2), assigned as $\gamma$-phase crystals of PVDF-TrFE. The dipole moment of $\gamma$-phase with TTTG' conformation has dipole moment slightly less than $\beta$-phase. Nonetheless, it does significantly contribute to the electrical properties of PVDF-TrFE film. Interestingly, peak at band $1290 \mathrm{~cm}^{-1}$ was shown to be less significant for UN and AN MEK (Fig. 2).

Nevertheless, upon dissolving PVDF-TrFE in DMF, a shift from $\gamma$-phase peak to $\beta$-phase peak was observed at similar wavenumber. The presence of $\beta$-phase at peak $1190 \mathrm{~cm}^{-1}$ was significant for UN DMF and AN DMF thin films. A sharp peak at 848 $\mathrm{cm}^{-1}$ was also observed for AN DMF, indicating significant presence of $\beta$-phase crystal in PVDF-TrFE.

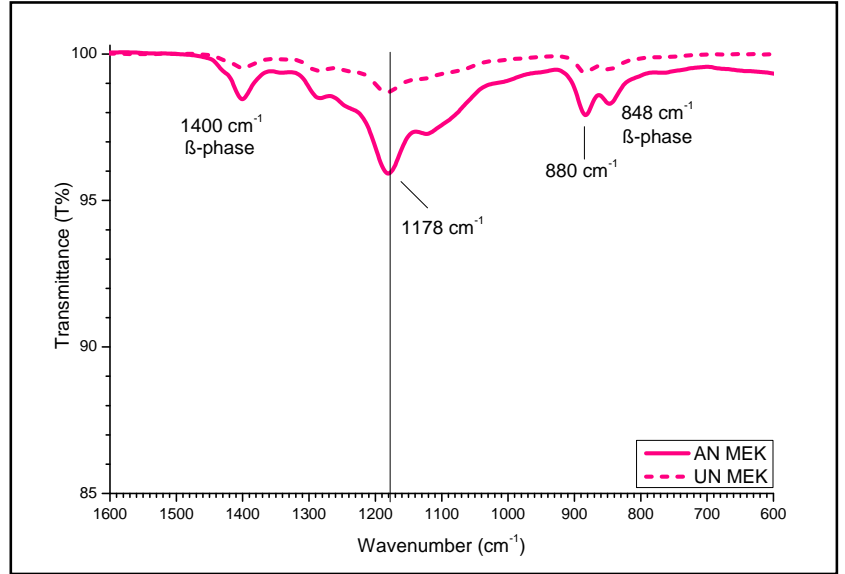

Fig. 2: ATR-FTIR spectra for the Un-annealed and annealed PVDF-TrFE thin film dissolved in MEK solvent

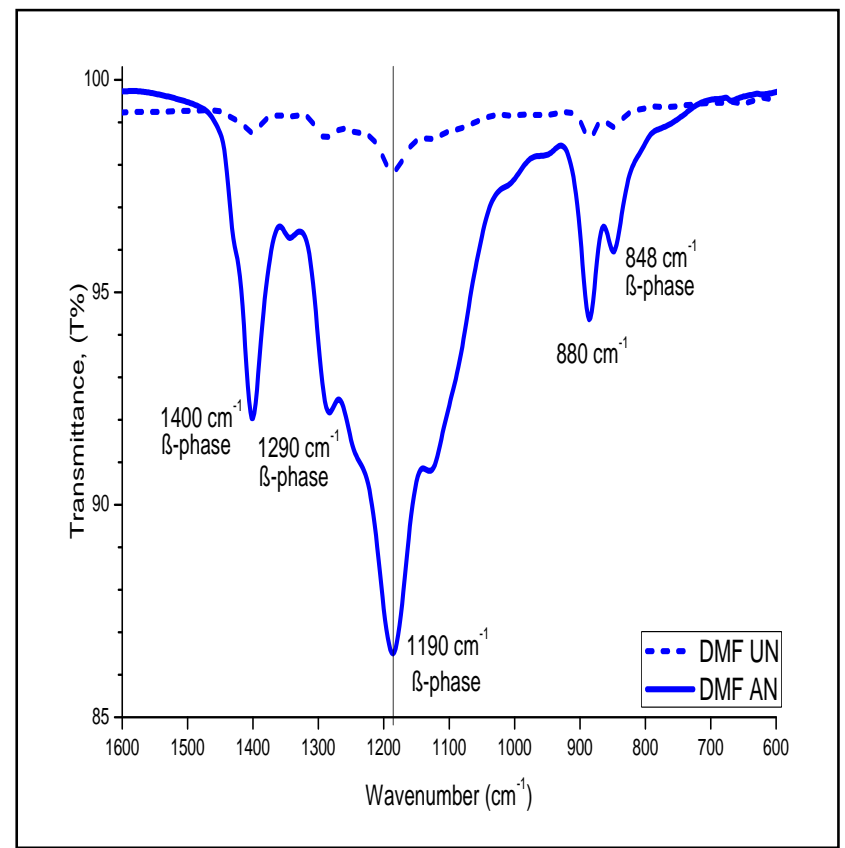

Fig. 3: ATR-FTIR spectra for the Un-annealed and annealed PVDF-TrFE thin film dissolved in DMF solvent.

\subsection{XRD}

The XRD patterns of un-annealed and annealed PVDF-TrFE thin films showed crystalline peak at angle $2 \theta=19.2^{\circ}$ (Fig. 4). This is equivalent to (200) and (110) planes. These peaks are assigned as $\beta$-crystal for PVDF-TrFE film [13, 15, 16]. Fig. 5 shows an overlay XRD spectra for cps values of unannealed and annealed PVDF-TrFE. The UN DEC, UN MEK and UN DMF values are within the range of 166 to 397 . However, significant increment in cps values was observed for all annealed PVDF-TrFE films. It also shows a $30 \%$ increment in cps value for $\mathrm{AN}$ DEC relative to UN DEC. The annealed PVDF-TrFE thin film formed from dissolution in MEK, showed a further increased in cps value (43\%). Amongst all films tested, annealed PVDF-TrFE thin film formed from dissolution in DMF showed highest cps value (53\%).

Interestingly, a small hump was observed in UN MEK at approximately $19^{\circ}$. This indicates the presence of PVDF-TrFE $\gamma$ phase crystals as supported by ATR-FTIR spectra (discussed earlier). Since $\gamma$-phase are also polar in origin, the cps value is greatly enhanced. Previous study reported by M.H Wahid et al., suggested that PVDF-TrFE in pellet that readily dissolved in MEK produced thin film with large cps value upon annealing at $120^{\circ} \mathrm{C}$ [13]. However, in this study, PVDF-TrFE powder dissolved in DMF produced thin film with the highest cps value. 


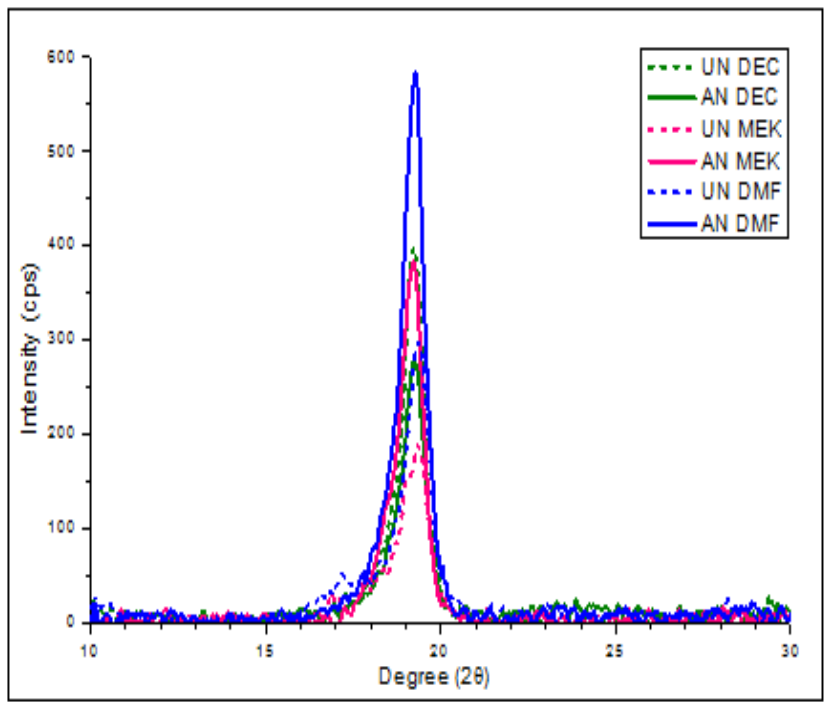

Fig. 4: XRD pattern for un-annealed and annealed PVDF-TrFE dissolve in DEC, MEK and DMF solvents

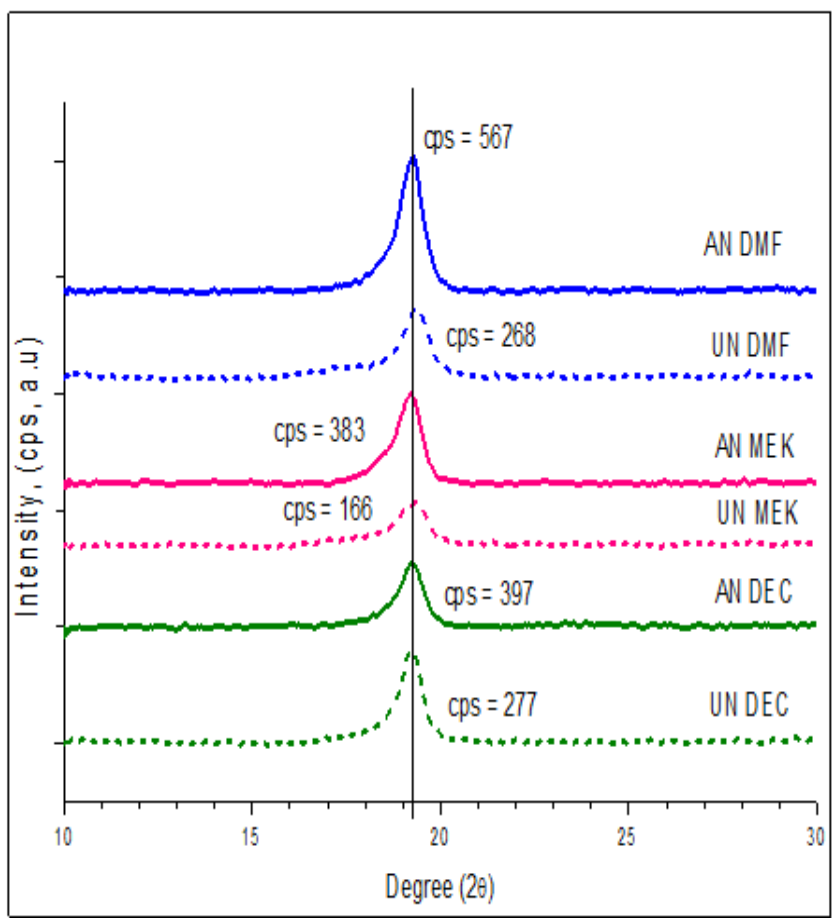

Fig. 5: Overlay of XRD pattern for un-annealed and annealed PVDF-TrFE thin films in DMF, MEK and DEC solvent with cps value.

\subsection{Solubility Parameters}

To determine the solubility parameters, the interaction radius $\left(\mathrm{R}_{0}\right)$ value is measured [17]. The value is determined from the radius of the sphere in Hansen space, which refers to three Hansen parameters as shown in Equation 1. The distance between Hansen parameters in Hansen space, (Ra), is calculated using the following formula:

$(\mathrm{Ra})^{2}=4(\delta \mathrm{d} . \mathrm{s}-\delta \text { d.p })^{2}+(\delta \mathrm{p} . \mathrm{s}-\delta \mathrm{p} \cdot \mathrm{p})^{2}+(\delta \mathrm{h} . \mathrm{s}-\delta \mathrm{h} . \mathrm{p})^{2}$

Where,

$\delta_{\text {d.s }}=$ Energy from dispersion forces of solvents,

$\delta_{\text {d.p }}=$ Energy from dispersion forces of polymer

$\delta_{\mathrm{p} . \mathrm{s}}=$ Energy from intermolecular forces of solvents

$\delta_{\mathrm{p} . \mathrm{p}}=$ Energy from intermolecular forces of polymer

$\delta_{\mathrm{p} . \mathrm{s}}=$ Energy from hydrogen bonds of solvents

$\delta_{\mathrm{p} . \mathrm{p}}=$ Energy from hydrogen bonds of polymer
Ratio of distance $(\mathrm{Ra})$ to interaction radius $\left(\mathrm{R}_{0}\right)$, the relative energy difference (RED) of the system is measured using the following formula;

$\mathrm{RED}=\mathrm{Ra} / \mathrm{R} 0$

Three conclusions were made from the RED value, which are: RED <1; complete dissolution of solute in solvent, RED = 1; partial dissolution of solute in solvent and RED > 1; insoluble of solute in solvent [17]. The RED values of PVDF-TrFE dissolved in DEC, MEK and DMF were obtained (Table 1).

Table 1: Hansen Solubility Parameters, Difference of Hansen solubility parameters with PVDF-TRFE and RED value for PVDF-TrFE, DEC, MEK and DMF solvents.

\begin{tabular}{c|c|c|c}
\hline Sample & $\begin{array}{c}\text { Hansen Solubility } \\
\text { Parameters } \\
(\delta \mathrm{t})(\mathrm{MPA})\end{array}$ & $\begin{array}{c}\text { Difference of } \delta \mathrm{t} \\
\text { with PVDF-TrFE }\end{array}$ & RED Value \\
\hline PVDF- & 23.1 & - & - \\
TrFE & 10.4 & 12.7 & 1.6 \\
DEC & 19.0 & 4.1 & 1.2 \\
MEK & 24.8 & 1.7 & 0.5 \\
DMF &
\end{tabular}

Hansen Solubility Parameter $(\delta \mathrm{t})$ value for PVDF-TrFE is 23.1 . DMF has $\delta$ t value of $24.8 \mathrm{MPa}$, suggesting a promising solvent for dissolving PVDF-TrFE. This is due to low difference in $\delta$ t value (between PVDF-TrFE and DMF). It is also evident from the visual clarity of the spin coated PVDF-TrFE film formed when utilizing DMF. This was further supported by ATR-FTIR spectra and XRD pattern. From the RED values, the DMF has lowest RED value in comparison to MEK and DEC. Belmares et al., and Fedors reported that RED value lower than 1.0 indicates a complete dissolution system [17, 18]. Hence, DMF is the suitable solvent required for dissolving PVDF-TrFE powder.

\subsection{Atomic Force Microscopy (AFM)}

Atomic Force Microscope was used to observe the surface morphology of PVDF-TrFE films dissolved in DEC, DMF and MEK, individually. Fig. 6 shows AFM images of $10 \mu \mathrm{m} \times 10 \mu \mathrm{m}$ (a) UN DEC, (b) AN DEC, (c) UN MEK, (d) AN MEK, (e) UN DMF, and (f) AN DMF films. The root-mean-square (RMS) for all films are tabulated in Table 1. All films showed a drop in the RMS values after annealing, indicating, reduction in the roughness of the annealed films. UN DEC and AN DEC (Fig. 6(a-b)) observed insignificant crystal structures and agglomerates. This can be related to the large difference in RED value between PVDF-TrFE powders and DEC solvent as discussed in section 3.3. The difference in the RMS values for UN DEC and AN DEC were also large, mainly due to the small difference in the boiling temperature of DEC $\left(\sim 126^{\circ} \mathrm{C}\right)$ as compared to the annealed temperature $\left(120^{\circ} \mathrm{C}\right)$. This may result in quick evaporation of the solvent, which impedes the formation of crystallite.

On the contrary, UN MEK, and AN MEK, showed relatively significant crystallite structures as observed in Fig. 6 (c-d). For UN MEK, a significant grain boundary can be observed, similar to the crystallites formed in PVDF-TrFE as reported by Wahid et al. [13]. However, upon annealing, the crystallites dispersed and formed smooth surfaces. This was supported by reduction of the RMS values (3.60) with comparison to the UN MEK (16.0).

Amongst all films observed, UN DMF and AN DMF showed a highly dense uniform crystallite structures. The presence of smaller grain boundaries can also be observed for both films.

Table 2: RMS value for UN DEC, AN DEC, UN MEK, AN MEK, UN DMF, and AN DMF

\begin{tabular}{cc}
\hline Films & RMS,,$(\mathbf{n m})$ \\
\hline UN DEC & 54.7 \\
AN DEC & 4.40 \\
UN MEK & 16.0 \\
AN MEK & 3.60 \\
UN DMF & 73.90 \\
AN DMF & 49.60 \\
\hline
\end{tabular}




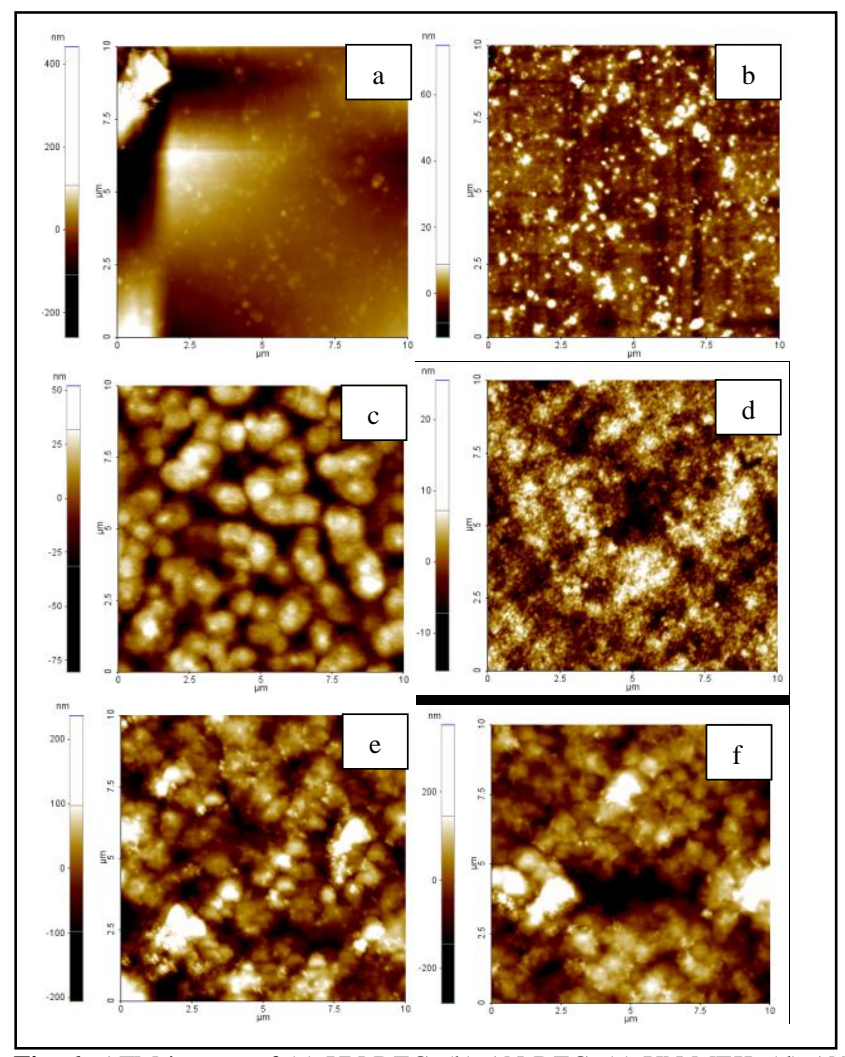

Fig. 6: AFM images of (a) UN DEC, (b) AN DEC, (c) UN MEK, (d) AN MEK, (e) UN DMF, and (f) AN DMF films.

\section{Conclusion}

Spin coated PVDF-TrFE thin films of $250 \mathrm{~nm}$ were prepared by solution casting using polar solvents of DEC, MEK and DMF. Annealed PVDF-TrFE thin films dissolved in DMF was found to produce improved $\beta$-phase crystals, as evident by FTIR spectrum; with significant absorption peaks at wavenumber of 848, 1190, and $1290 \mathrm{~cm}-1$. This finding is consistent with the high cps value obtained from the XRD pattern for AN DMF; an increment of $53 \%$ compared to UN DMF film. The RED value $(<1)$ suggests that DMF is an appropriate polar solvent for dissolving PVDFTrFE powders. Moreover, the crystallites formed are dense and uniform for both films, before and after annealing.

\section{Acknowledgement}

This research was fully supported by BESTARI Grant, IRMI Universiti Teknologi MARA (UiTM). The authors wish to acknowledge Faculty of Applied Sciences (FSG), Microwave Technology Institute (MTI), Faculty of Electrical Engineering (FKE), NANO-SciTech Centre (NST) and Institute of Science, UiTM Shah Alam for the XRD, ATR-FTIR and thermal evaporator equipment.

\section{References}

[1] Gan, W.C., W.H.A. Majid, and T. Furukawa, (2016) Ferroelectric polarization, pyroelectric activity and dielectric relaxation in Form IV poly(vinylidene fluoride). Polymer 82 156-165.

[2] Bae, J.-H. and S.-H. Chang, 2015 Characterization of an electroactive polymer (PVDF-TrFE) film-type sensor for health monitoring of composite structures. Composite Structures 131 1090-1098.

[3] Meng, N., Mao, Rui., Tu, Wei., Zhu, Xiaojing., Wilson, Rory M., Bilotti, Emiliano., and Reece, Michael J., (2016) Processing and characterization of free standing highly oriented ferroelectric polymer films with remarkably low coercive field and high remnant polarization. Polymer 100 69-76.

[4] Tan, Z., Fu, C., Gao, Y., Qian, J., Li, W., Wu, X., Chu, H., Chen, C., Nie, W., and Ran, X., (2018) Modifications of Gamma poly (vinylidene fluoride) ( $\gamma$-PVDF) films by high-energy electron beam irradiation. Radiation Physics and Chemistry, In Press.

[5] Nan, J., H., Qian, S., Jing, X., Guangmei, and S., Rui., (2017) Crystallization behavior and electroactive properties of PVDF, P (VDF-TrFE) and their blend films. Polymer Testing 57 302-306.

[6] Dhevi, D.M., A.A. Prabu, and K.J. Kim, (2018) Infrared spectroscopic studies on crystalline phase transition of PVDF and $\mathrm{PVDF} /$ hyperbranched polyester blend ultrathin films. Vibrational Spectroscopy 94 74-82.

[7] Chen, H.J., Han, S., Liu, C., Luo, Z., Shieh, H.P.D., Hsiao, R.S., and Yang, B.R., (2016) Investigation of PVDF-TrFE composite with nanofillers for sensitivity improvement. Sensors and Actuators A: Physical 245 135-139.

[8] Lee, J. and S. Lim, (2018) Polarization behavior of polyvinylidene fluoride films with the addition of reduced graphene oxide. Journal of Industrial and Engineering Chemistry, In Press.

[9] Gutiérrez, J., Martins, P., Gonçalves, R., Sencadas, V., Lasheras, A. Lanceros-Mendez, S., and Barandiarán, J. M., Synthesis, (2015) Physical and magnetic properties of BaFe12O19/P(VDF-TrFE) multifunctional composites. European Polymer Journal, 69 224-231.

[10] Tao, M.M., Liu, F., Ma, B.R., and Xue, L.X., (2013) Effect of solvent power on PVDF membrane polymorphism during phase inversion. Desalination 316 137-145.

[11] Bottino, A., Capannelli, G., Munari, S., and Turturro, A., (1988) Solubility parameters of poly (vinylidene fluoride). Journal of Polymer Science Part B: Polymer Physics 26 785-794

[12] Gregorio Jr, R. and D.S. Borges, (2008) Effect of crystallization rate on the formation of the polymorphs of solution cast poly(vinylidene fluoride). Polymer 49 4009-4016.

[13] Wahid, M.H., Rozana, M.D., Sa'ad, S.Z., Arshad, A.N., Sarip, M.N. Mahmood, M.R., Gan, W.C., Majid and W.C. Gan, and Majid, W.H.A., (2014) Ferroelectric Properties of PolyvinylidenefluorideTrifluoroethylene (PVDF-TrFE) Annealed Thin Film. Advanced Materials Research, 879 1-6.

[14] Mahdi, R.I., Gan, W. C., Abd Majid, W. H., Mukri, N.I., and Furukawa, T., (2018) Ferroelectric polarization and pyroelectric activity of functionalized $\mathrm{P}(\mathrm{VDF}-\mathrm{TrFE})$ thin film lead free nanocomposites. Polymer 141 184-193.

[15] Wahid, M.H., Rozana, M.D., Sa'ad, S.Z., Arshad, A.N., Sarip M.N., Habibah, Z., Rusop, M.R. (2015) Surface Morphologies of PVDFTrFE/MgO Nanocomposite Thin Films and its Effect on the Ferroelectric Properties. in Advanced Materials Research 1134 6-11.

[16] Cho, K.Y., Park, H., Kim, H.J., Do, X.H., Koo, C.M., Hwang, S.S., Yoon, H.G., and Baek, K.Y., (2018) Highly enhanced electromechanical properties of PVDF-TrFE/SWCNT nanocomposites using an efficient polymer compatibilizer. Composites Science and Technology 157 21-29.

[17] Belmares, M., Blanco, M., Goddard, W.A., Ross, R.B., Caldwell, G., Chou, S.H., Pham, J., Olofson, P.M. and Thomas, C., (2004) Hildebrand and Hansen solubility parameters from molecular dynamics with applications to electronic nose polymer sensors. Journal of computational chemistry 25 1814-1826.

[18] Fedors, R.F., (1974) A method for estimating both the solubility parameters and molar volumes of liquids. Polymer Engineering \& Science 14 147-154. 